\title{
CONGRUENCES ON AN ORTHODOX SEMIGROUP VIA THE MINIMUM INVERSE SEMIGROUP CONGRUENCE
}

\author{
by CARL EBERHART and WILEY WILLIAMS
}

(Received 1 April, 1976)

It is well known that the lattice $\Lambda(S)$ of congruences on a regular semigroup $S$ contains certain fundamental congruences. For example there is always a minimum band congruence $\beta$, which Spitznagel has used in his study of the lattice of congruences on a band of groups [16]. Of key importance to his investigation is the fact that $\beta$ separates congruences on a band of groups in the sense that two congruences are the same if they have the same meet and join with $\beta$. This result enabled him to characterize $\theta$-modular bands of groups as precisely those bands of groups for which $\rho \rightarrow(\rho \vee \beta, \rho \wedge \beta)$ is an embedding of $\Lambda(S)$ into a product of sublattices.

In this paper we make use of the minimum inverse semigroup congruence $\delta$ to investigate the congruences on an orthodox semigroup $S$. In Section 2 we show that $\phi(\rho)=\rho \vee \delta$ is a complete lattice homomorphism. In Section 3 we prove that $\delta$ separates congruences on $S$ in the sense that two congruences are the same if they have the same meet and join with $\delta$. Moreover if $\Phi$ is the congruence on $\Lambda(S)$ determined by the homomorphism $\phi$ above, then the $\Phi$-classes of $\Lambda(S)$ cross-section the $\theta$-classes in the sense that two congruences are the same if they lie in the same $\theta$ - and $\Phi$-classes. Using these results we show that $\Lambda(S)$ can be (lattice) embedded in $\Lambda(S) / \theta \times \Lambda(S / \delta)$ and that if $S$ is $\Phi$-modular, $\Upsilon(\rho)=(\rho \wedge \delta, \rho \vee \delta)$ is an embedding.

In Section 4 we investigate the structure of the product of a $\theta$-class with a $\Phi$-class, and give a sufficient condition under which $\Lambda(S)$ can be decomposed into a disjoint union of convex sublattices, each of which is isomorphic to a product of a $\theta$-class and a $\Phi$-class.

Applications of these results are collected in Section 5. We show that two congruences on an orthodox semigroup are equal if they have the same kernel and same $\theta$-class, extending a result of Scheiblich [15] for inverse semigroups. It is also shown that on a regular semigroup the lattice of inverse semigroup congruences is $\theta$-saturated. This is used to extend many of the results of Section 3 to the lattice $\Omega(S)$ of orthodox congruences on a regular semigroup.

1. Notation and preliminary results. Our notation follows Clifford and Preston [1]. $S$ denotes a regular semigroup, $E$ its set of idempotents and $\Lambda(S)$ the lattice of congruences on $S$. For $a \in S$,

$$
V(a)=\{x \in S: a x a=a \text { and } \quad x a x=x\} .
$$

Green's relations are denoted by $\mathscr{R}, \mathscr{L}, \mathscr{H}$ and $\mathscr{D}$, and the $\mathscr{H}$-class of $a$ by $H_{a} . \quad S$ will be called orthodox if $E$ is a subsemigroup.

RESULT 1.1. [12.] A congruence $\rho \in \Lambda(S)$ is completely determined by its idempotent classes; that is, if $\rho, \tau \in \Lambda(S)$, then $\rho=\tau$ if and only if, for each $e \in E, x \rho e \Leftrightarrow x \tau e$.

We denote the union of these idempotent classes by $\operatorname{ker} \rho=\{x \in S: x \rho e$ for some $e \in E\}$.

Glasgow Math. J. 18 (1977) 181-192. 
RESULT 1.2. [13.] For a regular semigroup $S$, the following are equivalent:

(i) $S$ is orthodox.

(ii) For each $a, b \in S$ and each $a^{\prime} \in V(a)$ and $b^{\prime} \in V(b), b^{\prime} a^{\prime} \in V(a b)$.

(iii) For each $e \in E, V(e) \subseteq E$.

Also in [13], Reilly and Scheiblich defined the relation $\theta$ on $\Lambda(S)$ by

$$
\rho \theta \tau \Leftrightarrow \rho \cap(E \times E)=\tau \cap(E \times E) .
$$

Their results, together with those of Scheiblich [14] and Hall [4], yield the following.

RESULT 1.3. The relation $\theta$ is a congruence on $\Lambda(S)$, each $\theta$-class is a complete modular sublattice, and the natural homomorphism of $\Lambda(S)$ onto $\Lambda(S) / \theta$ is a complete lattice homomorphism. Moreover, if $m(\rho)$ denotes the minimum of the $\theta$-class $\rho \theta$, this natural homomorphism is an isomorphism of $\rho \theta$ onto the lattice of idempotent-separating congruences on $\operatorname{S/m}(\rho)$.

RESULT 1.4. (Lallement [9].) If $\rho \in \Lambda(S)$, the idempotents of $S / \rho$ are precisely the elements of the form eo for some e $\in E$.

For a class $\mathscr{C}$ of semigroups, $\rho \in \Lambda(S)$ will be called a $\mathscr{C}$ congruence if $S / \rho \in \mathscr{C}$. In [7], Howie and Lallement studied the lattice properties of $\Lambda(S)$, and in particular the relationships between the minimum $\mathscr{C}$ congruences for various classes $\mathscr{C}$ of semigroups. We adopt the following symbols for certain of these:

$\mu=$ the maximum idempotent-separating congruence;

$\boldsymbol{\delta}=$ the minimum inverse semigroup congruence;

$\mathbf{1}_{\mathrm{s}}=$ the universal congruence $S \times S$;

$\mathbf{0}_{S}=$ the diagonal congruence $\{(x, x) \mid x \in S\}$;

$\lambda=$ the minimum orthodox semigroup congruence;

$\sigma=$ the minimum group congruence.

We also denote the lattice of orthodox congruences by $\Omega(S)=\{\rho \in \Lambda(S): \lambda \leqq \rho\}$ and the lattice of inverse semigroup congruences by $I(S)=\{\rho \in \Lambda(S): \delta \leqq \rho\}$. To denote one of the above congruences, say $\delta$, on another semigroup, say $T$, we use the notation $\delta(T)$. If $\tau \in \Lambda(S)$, then $\Lambda(\tau)$ denotes $\{\rho \in \Lambda(S): \rho \leqq \tau\}$. If $\alpha \leqq \beta$ in $\Lambda(S),[\alpha, \beta]=\{\gamma \in \Lambda(S): \alpha \leqq \gamma \leqq \beta\}$.

RESULT 1.5. (Hall [3].) If $S$ is orthodox, then $\delta=\{(x, y): x$ and $y$ have a common inverse $\}$. Moreover for each $e \in E, e \delta=V(e)$.

Let $S$ be an arbitrary semigroup. If $\rho, \gamma \in \Lambda(S)$ and $\gamma \leqq \rho$, then the relation $\rho / \gamma$ on $S / \gamma$ defined by $\rho / \gamma=\{(x \gamma, y \gamma):(x, y) \in \rho\}$ is a congruence. Moreover the lattice $\Lambda(S) \vee \gamma$ is isomorphic with $\Lambda(S / \gamma)$ under the map $\gamma \vee \tau \rightarrow(\gamma \vee \tau) / \gamma$. In particular, if $\gamma \leqq \rho, \tau$, then $(\rho \wedge \tau) / \gamma=(\rho / \gamma) \wedge(\tau / \gamma)$ and $(\rho \vee \tau) / \gamma=(\rho / \gamma) \vee(\tau / \gamma)$. If $S$ is also regular, then it follows from Result 1.4 that if $\gamma \leqq \rho, \tau$ then $\rho \theta \tau \Leftrightarrow \rho / \gamma \theta \tau / \gamma$. These results are easily verified, as is pointed out in [13] and [16].

If $\rho$ is a relation on $S$ then $\rho^{*}$ denotes the congruence generated by $\rho$.

2. The mapping $\rho \rightarrow \rho \vee \delta$. Throughout the next three sections $S$ denotes an orthodox semigroup and $p \in \Lambda(S)$. 
LEMMA 2.1. If $x(\rho \vee \delta)$ e for some $e \in E$, then $x \rho f$ for some $f \in E$. Thus $\operatorname{ker}(\rho)=\operatorname{ker}(\rho \vee \delta)$.

Proof. Suppose that $x(\rho \vee \delta) e$, where $e \in E$. It suffices by Result 1.4 to show $x \rho x^{2}$. Let $y \in V(x)$. Since $\rho \vee \delta$ is an inverse semigroup congruence, we have $y(\rho \vee \delta) e$. Also $x y$ is an idempotent $f$ in $R_{x}$, and $x(\rho \vee \delta) e(\rho \vee \delta) x e(\rho \vee \delta) x y=f$. Thus $f x f(\rho \vee \delta) f^{3}=f$. We shall show that $f x f \rho f$, for then $x^{2}=(f x)^{2}=f x f x \rho f x=x$, and we will be done. Since $f x f(\rho \vee \delta) f$, there exist $x_{1}, x_{2}, \ldots, x_{n}$ in $S$ such that $f \rho x_{1} \delta x_{2} \rho \ldots \rho x_{n} \delta f x f$. Setting $y_{i}=f x_{i} f$ one computes that $f \rho y_{1} \delta y_{2} \rho \ldots \rho y_{n} \delta f x f$. By Result 1.5, $y_{1}$ and $y_{2}$ have a common inverse $w$, and since $y_{1}, y_{2} \in f S f, f w f$ is also a common inverse. Now $f w f=f(f w f) f \rho y_{1}(f w f) y_{1}=y_{1} \rho f$, so $f w f \rho f$. Also $y_{2}=f y_{2} f \rho(f w f) y_{2}(f w f)=f w f \rho f$, so $y_{2} \rho f$. Continuing in this manner, we have $f \rho f x f$, and the proof is complete.

\section{Lemma 2.2. Let $e, f \in E$. Then $e(\rho \vee \delta) f$ iff $e \rho$ efe and $f \rho f e f$.}

Proof. If $e(\rho \vee \delta) f$, then $e=e^{3}(\rho \vee \delta) e f e$. Thus there exist $x_{1}, \ldots, x_{n}$ such that $e \rho x_{1} \delta x_{2} \ldots \rho x_{n} \delta e f e$. Without loss of generality these may be chosen in $e S e$, and proceeding as in 2.1 we have $e \rho$ efe. Similarly $f \rho f e f$.

Conversely suppose $e \rho$ efe and $f \rho f e f$. Since $e f \delta f e$, we have $f e f \delta f e$ and $e f \delta e f e$. Thus $e \rho$ efe $\delta$ ef $\rho$ ef $\delta f e \rho f e \delta f e f \rho f$, and $e(\rho \vee \delta) f$.

THEOREM 2.3. The minimum inverse semigroup congruence on $S / \rho$, denoted $\delta(S / \rho)$, equals $(\rho \vee \delta) / \rho$.

Proof. First note that $\delta(S / \rho) \leqq(\rho \vee \delta) / \rho$ since $(\rho \vee \delta) / \rho$ is an inverse semigroup congruence on $S / \rho$. . Define $\tau \in \Lambda(S)$ by $x \tau y \Leftrightarrow(x \rho) \delta(S / \rho)(y \rho)$. Then $\tau \geqq \rho$, and $\tau / \rho=\delta(S / \rho)$. Also $\tau \geqq \delta$ since $S / \tau=(S / \rho) /(\tau / \rho)=(S / \rho) / \delta(S / \rho)$. Hence $\tau \geqq \rho \vee \delta$ and so $\tau / \rho=\delta(S / \rho) \geqq$ $(\rho \vee \delta) / \rho$. This establishes the equality.

We remark here that Theorem 2.3 holds more generally for any minimum $\mathscr{C}$ congruence provided $\mathscr{C}$ is closed under homomorphic images.

THEOREM 2.4. If $\mathscr{A}$ is a collection of congruences on $S$, then $\delta \vee(\cap \mathscr{A})=\cap\{\delta \vee \rho: \rho \in \mathscr{A}\}$. Hence the function $\phi(\rho)=\rho \vee \delta$ is a complete lattice homomorphism from $\Lambda(S)$ onto $I(S)$.

Proof. Clearly $\delta \vee(\cap \mathscr{A}) \subseteq \cap\{\delta \vee \rho: \rho \in \mathscr{A}\}$. To show the converse suppose $x(\cap\{\delta \vee \rho: \rho \in \mathscr{A}\}) e$. Then $x(\delta \vee \rho) e$ for all $\rho \in \mathscr{A}$, so, by $2.1, x \rho f_{\rho}$ for some $f_{\rho} \in E$. This implies $e(\rho \vee \delta) f_{\rho}$ and thus by Lemma 2.2 we have $e f_{\rho} e \rho e$ and $f_{\rho} e f_{\rho} \rho f_{\rho}$ for all $\rho \in \mathscr{A}$. We deduce that exe $\rho e$ and $x \operatorname{ex} \rho x$ for all $\rho \in \mathscr{A}$, so exe $(\cap \mathscr{A}) e$ and $x \operatorname{ex}(\cap \mathscr{A}) x$. By Result 1.5, this means $x(\cap \mathscr{A})$ and $e(\cap \mathscr{A})$ are related by $\delta(S / \cap \mathscr{A})$. Since $(\delta \vee(\cap \mathscr{A})) / \cap \mathscr{A}$ is an inverse semigroup congruence on $S / \cap \mathscr{A}$, we have $x(\cap \mathscr{A})$ and $e(\cap \mathscr{A})$ related by $(\delta \vee(\cap \mathscr{A})) / \cap \mathscr{A}$, and thus $x(\delta \vee(\cap \mathscr{A})) e$. By Result 1.1 we are done.

Combining this with the remarks at the end of Section 1 we have the following result.

COROLLARY 2.5. The function $\rho \rightarrow(\rho \vee \delta) / \delta$ is a complete lattice homomorphism of $\Lambda(S)$ onto $\Lambda(S / \delta)$.

3. Embeddings of $\Lambda(S)$ in product lattices, A congruence $\rho$ is idempotent separating if $\rho \theta \mathbf{0}_{\mathrm{S}}$. This idea was first considered by Munn [11] who showed that these are precisely the 
congruences contained in $\mathscr{H}$. Munn also showed that these congruences commute with each other. The next lemma shows that they also commute with any congruence below $\delta$. One should note, however, that congruences below $\delta$ need not commute with each other.

LeMma 3.1. If $\rho, \tau \in \Lambda(S)$ with $\rho \subseteq \mu$ and $\tau \subseteq \delta$, then $\rho \circ \tau=\tau \circ \rho=\rho \vee \tau$.

Proof. Suppose $x \rho z \tau y$. Then $x \mu z \delta y$, so there exists $w$ in $V(y) \cap V(z)$. One computes that $y=y w y=y w z w y \rho y w x w y$. Also $x=z w x w z$ since $z w$ and $w z$ are $\mathscr{R}$ - and $\mathscr{L}$-related to $z$ (and hence to $x$ ), respectively. But $z w x w z \tau y w x w y$, so $x \tau y w x w y \rho y$ and $x(\tau \circ \rho) y$, and we are done.

LEMMA 3.2. If $\rho, \tau \subseteq \mu$ and $\rho \vee \delta=\tau \vee \delta$ then $\rho=\tau$.

Proof. Suppose $\rho, \tau \subseteq \mu$ and $\rho \vee \delta=\tau \vee \delta$. Then $\rho \circ \delta=\rho \vee \delta=\tau \vee \delta=\tau \circ \delta$. We show that $x \rho e$ implies $x \tau e$. If $x \rho e$ then $x(\rho \circ \delta) e$ so $x(\tau \circ \delta) e$. Thus there exists $y$ such that $x \tau y \delta e$. However $x \rho e$ and $\rho \subseteq \mu \subseteq \mathscr{H}$ imply that $e$ is the identity of $H_{x}$, and $y \delta e$ implies $y \in E$. But then $x \tau y$ and $\tau \subseteq \mathscr{H}$ imply $y$ is the identity of $H_{x}$, so $y=e$ and $x \tau e$. By interchanging the roles of $\rho$ and $\tau$ we see that $x \rho e$ iff $x \tau e$, and we are done.

Lemma 3.3. If $\rho \theta \tau$ and $\rho \vee \delta=\tau \vee \delta$, then $\rho=\tau$.

Proof. Let $\kappa$ be the least element of $\rho \theta$. Then $\rho / \kappa$ and $\tau / \kappa$ are idempotent separating on $S / \kappa$. If $\rho \vee \delta=\tau \vee \delta$, then, by Theorem 2.3, $(\rho / \kappa) \vee \delta(S / \kappa)=(\rho / \kappa) \vee((\kappa \vee \delta) / \kappa)=$ $(\rho \vee \kappa \vee \delta) / \kappa=(\rho \vee \delta) / \kappa=(\tau \vee \delta) / \kappa=(\tau / \kappa) \vee \delta(S / \kappa)$. By Lemma 3.2, this implies $\rho / \kappa=\tau / \kappa$, which implies $\rho=\tau$.

THEOREM 3.4. If $\rho \vee \delta=\tau \vee \delta$ and $\rho \wedge \delta=\tau \wedge \delta$ then $\rho=\tau$.

Proof. . Assume $\rho \vee \delta=\tau \vee \delta$ and $\rho \wedge \delta=\tau \wedge \delta$. By Lemma 3.3 it suffices to show $\rho \theta \tau$. If $e \rho f$ then $e(\rho \vee \delta) f$, and so $e(\tau \vee \delta) f$. By Lemma 2.2 this means $e \rho e f e, f \rho f e f$, $e \tau e f e$, and $f \tau f e f$. Also efe $\delta f e f$ since idempotents commute in $S / \delta$, and efe $\rho f e f$ since $e \rho f$. Thus efe $(\rho \wedge \delta) f e f$, so $e f e(\tau \wedge \delta) f e f$ and hence efe $\tau f e f$. Combining these results we have $e \tau f$. Interchanging $\rho$ and $\tau$ yields $\rho \theta \tau$, and the proof is complete.

Combining this theorem with Corollary 2.5 we have the following result.

Corollary 3.5. (a) The function $\rho \rightarrow(\rho \theta,(\rho \vee \delta) / \delta)$ from $\Lambda(S)$ to $\Lambda(S) / \theta \times \Lambda(S / \delta)$ is a complete lattice embedding.

(b) The function $Y(\rho)=(\rho \wedge \delta, \rho \vee \delta)$ is one-to-one.

The function $\Upsilon$ in (b) is not in general a lattice homomorphism, for $\delta \wedge(\rho \vee \tau)$ may not equal $(\delta \wedge \rho) \vee(\delta \vee \tau)$, even in bands (where $\delta=\mathscr{D})$. Consider the following example.

EXAMPLE 3.6. Let $D_{1}$ and $D_{2}$ be right zero bands on $\{e, f\}$ and $\left\{e^{\prime}, f^{\prime}\right\}$ respectively. Let $\phi: D_{1} \rightarrow D_{2}$ be defined by $\phi(e)=e^{\prime}$ and $\phi(f)=f^{\prime}$, and define $B$ to be the mapping band on $D_{1} \cup D_{2}$. Thus for $a \in D_{1}$ and $b \in D_{2}, a b=\phi(a) b=b$ and $b a=b \phi(a)=\phi(a)$.

$\Lambda(B)$ has three elements other than $\mathbf{1}$ and $\mathbf{0}$. One is $\delta=\mathscr{D}$. Another is $\rho=\left\{\left(e^{\prime}, f^{\prime}\right)\right\}^{*}$ which has only one nontrivial class, namely $\left\{e^{\prime}, f^{\prime}\right\}$. The last is $\tau=\left\{\left(e, e^{\prime}\right)\right\}^{*}$ which has only two nontrivial classes, $\left\{e, e^{\prime}\right\}$ and $\left\{f, f^{\prime}\right\}$. It is easily computed that $e(\delta \wedge(\rho \vee \tau)) f$, however $e[(\rho \wedge \delta) \vee(\tau \wedge \delta)] f$ is false. The complete lattice of congruences on $B$ is as in Figure 1. 


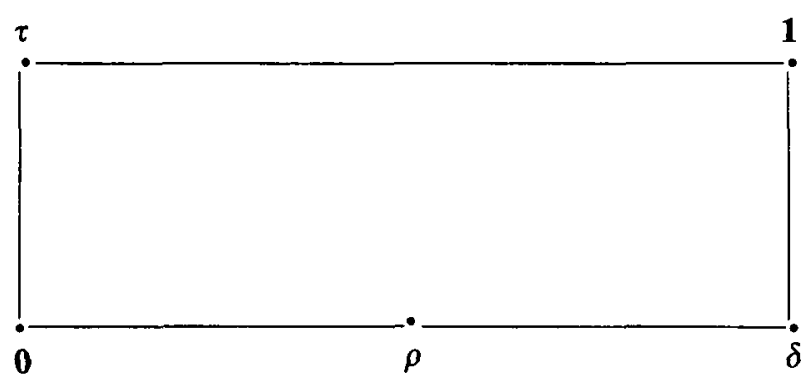

FIGURE 1

In [16], Spitznagel made the following definition. Let $\Gamma$ be a congruence on $\Lambda(S)$. We say that $\Lambda(S)$ is $\Gamma$-modular provided the conditions $\rho, \tau, \alpha \in \Lambda(S), \rho \leqq \tau, \rho \Gamma \tau, \alpha \vee \rho=\alpha \vee \tau$, and $\alpha \wedge \rho=\alpha \wedge \tau$ imply $\rho=\tau$. Spitznagel then showed that if $T$ is a band of groups, that is, a union of groups on which $\mathscr{H}$ is a congruence, and if $\beta$ is the minimum band congruence on $T$, then the function $\psi: \Lambda(T) \rightarrow(\Lambda(T) \vee \beta) \times(\Lambda(T) \wedge \mu)$ defined by $\psi(\rho)=(\rho \vee \mathscr{H}, \rho \wedge \mathscr{H})$ is an embedding if and only if $\Lambda(S)$ is $\theta$-modular.

Define the relation $\Phi$ on $\Lambda(S)$ by $\rho \Phi \tau \Leftrightarrow \rho \vee \delta=\tau \vee \delta$. If $S$ is an orthodox semigroup, then, by Theorem 2.4, $\Phi$ is the congruence associated with the homomorphism $\phi(\rho)=\rho \vee \delta$, and the $\Phi$-classes are complete convex sublattices of $\Lambda(S)$. Moreover it follows from Lemma 3.3 that the $\Phi$-classes cross-section the $\theta$-classes of $\Lambda(S)$ in the sense that $\rho \theta \tau$ and $\rho \Phi \tau$ imply $\rho=\tau$. We can now prove an analogue of part of Spitznagel's result.

THEOREM 3.7. If $\Lambda(S)$ is $\Phi$-modular, then the map $\Upsilon(\rho)=(\rho \wedge \delta, \rho \vee \delta)$ is an embedding of $\Lambda(S)$ into $\Lambda(\delta) \times I(S)$

Proof. From Corollaries 2.5 and 3.5 we need only show that $\rho \rightarrow \rho \wedge \delta$ is $\vee$-preserving; that is, $(\rho \vee \tau) \wedge \delta=(\rho \wedge \delta) \vee(\tau \wedge \delta)$. We note that $(\rho \wedge \delta) \vee(\tau \wedge \delta) \leqq(\rho \vee \tau) \wedge \delta$, and that since both are below $\delta$, they are $\Phi$-related. Thus since $\Lambda(S)$ is $\Phi$-modular, in order to show $(\rho \vee \tau) \wedge \delta=(\rho \wedge \delta) \vee(\tau \wedge \delta)$ it suffices to show that $(1) \rho \vee[(\rho \vee \tau) \wedge \delta]=\rho \vee[(\rho \wedge \delta) \vee$ $(\tau \wedge \delta)]$, and $(2) \rho \wedge[(\rho \vee \tau) \wedge \delta]=\rho \wedge[(\rho \wedge \delta) \vee(\tau \wedge \delta)]$.

For the first equality, note first that $(\rho \wedge \delta) \vee(\tau \wedge \delta) \leqq(\rho \vee \tau) \wedge \delta$ and hence $\rho \vee$ $[(\rho \wedge \delta) \vee(\tau \wedge \delta)] \leqq \rho \vee[(\rho \vee \tau) \wedge \delta]$. Now note that $\rho \vee[(\rho \wedge \delta) \vee(\tau \wedge \delta)]=\rho \vee(\tau \wedge \delta)$. Since $\delta \vee[\rho \vee[(\rho \vee \tau) \wedge \delta]]=(\rho \vee \delta) \vee[\delta \vee[(\rho \vee \tau) \wedge \delta]]=(\rho \vee \delta) \vee \delta=\rho \vee \delta=(\delta \vee \rho)$ $\vee(\tau \wedge \delta)=\delta \vee[\rho \vee(\tau \wedge \delta)]$, we have that $\rho \vee(\tau \wedge \delta) \Phi \rho \vee[(\rho \wedge \delta) \vee(\tau \wedge \delta)]$. Consequently, to show (1) it will suffice, by $\Phi$-modularity, to show that (a) $\tau \vee[\rho \vee$ $(\tau \wedge \delta)]=\tau \vee[\rho \vee[(\rho \wedge \delta) \vee(\tau \wedge \delta)]]$ and (b) $\tau \wedge[\rho \vee(\tau \wedge \delta)]=\tau \wedge[\rho \vee[(\rho \wedge \delta) \vee$ $(\tau \wedge \delta)]]$. For (a), note that $\tau \vee \rho \leqq \tau \vee[\rho \vee(\tau \wedge \delta)] \leqq \tau \vee[\rho \vee[(\rho \vee \tau) \wedge \delta]] \leqq \tau \vee[\rho \vee$ $(\rho \vee \tau)]=\tau \vee \rho$, so (a) holds. For (b), we first note $\tau \wedge[\rho \vee(\tau \wedge \delta)] \leqq \tau \wedge[\rho \vee[(\rho \vee \tau) \wedge \delta]]$ $\leqq \tau \wedge[\rho \vee \delta]$. If we can show (c) $\tau \wedge(\rho \vee \delta)=\tau \wedge[\rho \vee(\tau \wedge \delta)]$, we will be done. First note that, by Theorem 2.4, $\delta \vee[\tau \wedge(\rho \vee \delta)]=(\delta \vee \tau) \wedge[\delta \vee(\rho \vee \delta)]=(\delta \vee \tau) \wedge(\rho \vee \delta)=$ $(\delta \vee \tau) \wedge(\delta \vee \rho \vee(\tau \wedge \delta))=\delta \vee[\tau \wedge[\rho \vee(\tau \wedge \delta)]]$. Hence (c) will follow from Lemma 3.3 if we can show that $\tau \wedge(\rho \vee \delta) \theta \tau \wedge(\rho \vee(\tau \wedge \delta))$. If $e(\tau \wedge(\rho \vee \delta)) f$, then $e \tau f$ and $e(\rho \vee \delta) f$. By Lemma 2.2 we then have efe $\rho e$ and $f e f \rho f$. Also $e \tau f$ implies efe $\tau f e f$. Since $S$ 
is orthodox, one can easily compute $e f e \in V(f e f)$, and hence $e f e \delta f e f$ by Result 1.5. Thus $e \rho e f e(\tau \wedge \delta) f e f \rho f$, so $e(\rho \vee(\tau \wedge \delta)) f$. As noted above, $e \tau f$, so $e(\tau \wedge[\rho \vee(\tau \wedge \delta)]) f$. It now follows from $\tau \wedge(\rho \vee(\tau \wedge \delta)) \leqq \tau \wedge(\rho \vee \delta)$ that $\tau \wedge(\rho \vee \delta) \theta \tau \wedge(\rho \vee(\tau \wedge \delta))$, and the proof of (1) is complete.

For $(2)$, since $(\rho \wedge \delta) \vee(\tau \wedge \delta) \leqq \delta$ we have $\rho \wedge[(\rho \wedge \delta) \vee(\tau \wedge \delta)] \leqq \rho \wedge \delta$. However $\rho \wedge \delta=\rho \wedge(\rho \wedge \delta) \leqq \rho \wedge[(\rho \wedge \delta) \vee(\tau \wedge \delta)]$, and (2) follows. The proof is now complete.

The converse to this theorem is not true, and in this way the analogy between our situation and that of Spitznagel [16] breaks down. The reason for this is that while $\theta$-classes are modular [13], $\Phi$-classes need not be. The interested reader can check that if $B$ is the " $4 \times 4$ " rectangular band, then $\Lambda(B)$ has only one $\Phi$-class, and it is non-modular.

4. Decomposing $\Lambda(S)$ into a union of product lattices. We have seen that a congruence $\rho$ on an orthodox semigroup $S$ is completely determined by $\rho \wedge \delta$ and $\rho \vee \delta$. A natural question is the following: Given $\gamma \leqq \delta$ and $\tau \geqq \delta$, under what conditions does there exist a $\rho \in \Lambda(S)$ such that $\rho \wedge \delta=\gamma$ and $\rho \vee \delta=\tau$ ? Example 3.6 shows that in general such a $\rho$ need not exist.

In the sequel we shall denote the minimum element of $\rho \Phi$ by $\bar{\rho}$. It is not hard to show that $\overline{\mathbf{1}}_{S}$ is the minimum rectangular band congruence on $S$, and $\bar{\sigma}$ is the minimum completely simple semigroup (here rectangular group) congruence on $S$.

We now show that $\rho$ can be recovered from $\bar{\rho}$ and $\delta \wedge \rho$.

LEMMA 4.1. If $\rho \in \Lambda(S)$, then $\rho=(\delta \wedge \rho) \vee \bar{\rho}$.

Proof. Since $\delta \vee[(\delta \wedge \rho) \vee \bar{\rho}]=\delta \vee \bar{\rho}=\delta \vee \rho$, by Lemma 3.3 we need only show $\rho \theta(\delta \wedge \rho) \vee \bar{\rho}$. Always we have $\rho \geqq(\delta \wedge \rho) \vee \bar{\rho}$, so this reduces to showing that $e \rho f$ implies $e[(\delta \wedge \rho) \vee \bar{\rho}] f$. If $e \rho f$, then $e(\bar{\rho} \vee \delta) f$ and so $e \bar{\rho} e f e$ and $f \bar{\rho} f e f$ by Lemma 2.2. But $e \rho f$ also implies efe $\rho f e f$, and efe $\delta f e f$ since $\delta$ is an inverse semigroup congruence. Hence $e \bar{\rho} e f e(\rho \wedge \delta) f e f \bar{\rho} f$, so $e[(\delta \wedge \rho) \vee \bar{\rho}] f$.

THEOREM 4.2. Suppose $\gamma, \tau \in \Lambda(S)$ such that $\gamma \leqq \delta$ and $\tau \geqq \delta$. The following are equivalent:

(i) There exists $\rho \in \Lambda(S)$ such that $\rho \vee \delta=\tau$ and $\rho \wedge \delta=\gamma$.

(ii) $(\bar{\tau} \vee \gamma) \wedge \delta=\gamma$.

Proof. (i) $\Rightarrow$ (ii): Let $\rho$ be such that $\rho \vee \delta=\tau$ and $\rho \wedge \delta=\gamma$. Then $\rho \geqq \bar{\tau}$ and $\rho \geqq \gamma$, so $\rho \geqq \bar{\tau} \vee \gamma$. Hence $\gamma=\rho \wedge \delta \geqq(\bar{\tau} \vee \gamma) \wedge \delta \geqq \gamma$.

(ii) $\Rightarrow$ (i): Suppose $\gamma \leqq \delta$ and $\tau \geqq \delta$ are such that $(\bar{\tau} \vee \gamma) \wedge \delta=\gamma$. Setting $\rho=\bar{\tau} \vee \gamma$, one easily computes that $\rho \wedge \delta=\gamma$ and $\rho \vee \delta=\tau$.

Another natural problem is to investigate the sublattices of $\Lambda(S)$ consisting of the product of a $\theta$-class and a $\Phi$-class. The simplest of these is $\Lambda(\mu) \times \Lambda(\delta)$, and we begin with it.

LEMMA 4.3. If $\rho, \tau \leqq \mu \vee \delta$, then $\rho \theta \tau$ iff $\rho \wedge \delta=\tau \wedge \delta$. Thus each congruence below $\delta$ is the minimum element of its $\theta$-class.

Proof. It is easy to show that in general for $\alpha, \rho \in \Lambda(S), \alpha \leqq \rho$ implies $\bar{\alpha} \leqq \bar{\rho}$. Also $\overline{\mu \vee \delta}=\mu$, since $\mu$ is the least element of $(\mu \vee \delta) \Phi$. For any $\alpha \leqq \mu \vee \delta$ we then have $0_{S} \leqq \bar{\alpha} \leqq \mu$, 
so that $\bar{\alpha} \theta \mathbf{0}_{S}$. Since $\theta$ is a congruence, $\bar{\alpha} \vee(\alpha \wedge \delta) \theta \mathbf{0}_{S} \vee(\alpha \wedge \delta)=\alpha \wedge \delta$. But, by Lemma 4.1, $\alpha=\bar{\alpha} \vee(\alpha \wedge \delta)$, so $\alpha \theta \alpha \wedge \delta$.

Now if $\rho, \tau \leqq \mu \vee \delta$, then $\rho \wedge \delta=\tau \wedge \delta$ implies $\rho \theta \rho \wedge \delta=\tau \wedge \delta \theta \tau$, so $\rho \theta \tau$. Conversely, if $\rho \theta \tau$, then $\rho \wedge \delta \theta \tau \wedge \delta$. But $(\rho \wedge \delta) \vee \delta=\delta=(\tau \wedge \delta) \vee \delta$, so, by Lemma 3.3, $\rho \wedge \delta=\tau \wedge \delta$.

For the last statement, if $\alpha \leqq \delta$ then for any $\rho \in \alpha \theta$, consider $(\rho \wedge \alpha) \vee \delta$ and $(\rho \wedge \alpha) \wedge \delta$. Then $\rho \wedge \alpha=\rho \wedge(\alpha \wedge \delta)=(\rho \wedge \delta) \wedge \alpha=(\alpha \wedge \delta) \wedge \alpha=\alpha$, so $\alpha \leqq \rho$, and the proof is complete.

THEOREM 4.4. If $\rho, \tau \leqq \mu \vee \delta$, then $(\rho \vee \tau) \wedge \delta=(\rho \wedge \delta) \vee(\tau \wedge \delta)$. Consequently $\Lambda(\mu \vee \delta)$ is isomorphic to $\Lambda(\mu) \times \Lambda(\delta)$.

Proof. Suppose $\rho, \tau \leqq \mu \vee \delta$. By the above lemma, $[(\rho \vee \tau) \wedge \delta] \theta(\rho \vee \tau) \theta[(\rho \wedge \delta) \vee$ $(\tau \wedge \delta)]$, and since $(\rho \vee \tau) \wedge \delta$ and $(\rho \wedge \delta) \vee(\tau \wedge \delta)$ are in $\Lambda(\delta)$, they are equal.

Thus the function $\Upsilon(\rho)=(\rho \wedge \delta, \rho \vee \delta)$ of Corollary 3.5(b) is a lattice homomorphism of $\Lambda(\mu \vee \delta)$ into $\Lambda(\delta) \times[\delta, \mu \vee \delta]$. To show that it is onto, suppose $\gamma \in \Lambda(\delta)$ and $\tau \in[\delta, \mu \vee \delta]$. Then $(\bar{\tau} \vee \gamma) \wedge \delta=(\bar{\tau} \wedge \delta) \vee(\gamma \wedge \delta)=\mathbf{0}_{s} \vee \gamma=\gamma$, and so, by Theorem 4.2, there exists a $\rho \in \Lambda(\mu \vee \delta)$ such that $\rho \vee \delta=\tau$ and $\rho \wedge \delta=\gamma$. Thus $\Upsilon$ is onto $\Lambda(\delta) \times[\delta, \mu \vee \delta]$.

But the mapping $\rho \rightarrow \bar{\rho}$ of $[\delta, \mu \vee \delta]$ onto $\Lambda(\mu)$ is an isomorphism since it is the inverse of the mapping $\phi(\rho)=\rho \vee \delta$. Thus $\Lambda(\mu \vee \delta)$ is isomorphic to $\Lambda(\mu) \times \Lambda(\delta)$ under the mapping $\rho \rightarrow(\bar{\rho}, \rho \wedge \delta)$, and the proof is complete.

Outside $\Lambda(\mu \vee \delta)$ things are not so nice. We shall see below that although $\mu$ is the top of its $\theta$-class, $\mu \vee \delta$ need not be the top of its $\theta$-class. Also $\bar{\rho} \wedge \delta$ need not be $\mathbf{0}_{s}$, so $\Lambda(S)$ is not necessarily a product. However, one might hope to write it as a union of products of $\theta$-classes with sublattices of $\Lambda(\delta)$. This idea motivates the rest of this section.

First we would like to find a condition under which $\mu \vee \delta$ is the maximum element of its $\theta$-class. If $\tau$ and $\beta$ are, respectively, the maximum and minimum elements of a $\theta$-class of a regular semigroup $S$, Reilly and Scheiblich [13] have shown that $\rho \rightarrow \rho / \beta$ is an isomorphism of $\beta \theta$ onto the lattice of idempotent separating congruences on $S / \beta$, and that $\mu(S / \beta)=\tau / \beta$. In what follows we let $\beta=\delta$ and let $\tau$ be the largest element of $\delta \theta$, so

$$
a \tau b \Leftrightarrow(a \delta) \tau / \delta(b \delta) \Leftrightarrow(a \delta) \mu(S / \delta)(b \delta)
$$

J. M. Howie [6] has characterized $\mu$ on an inverse semigroup $T$ by

$$
\mu(T)=\left\{(x, y) \in T \times T: x e x^{-1}=y e y^{-1} \text { for all idempotents } e \in T\right\} .
$$

On an orthodox semigroup $S, \mu$ has been characterized by J. Meakin [10] as

$$
\begin{array}{r}
\mu(S)=\left\{(a, b) \in S \times S: \text { there exists } a^{\prime} \in V(a) \text { and } b^{\prime} \in V(b)\right. \text { for which } \\
\left.\qquad a e a^{\prime}=b e b^{\prime} \text { and } a^{\prime} e a=b^{\prime} e b \text { for all } e \in E\right\} .
\end{array}
$$

Thus for $\tau$ the maximum of $\delta \theta$, we have

$$
a \tau b \Leftrightarrow(a \delta) F(a \delta)^{-1}=(b \delta) F(b \delta)^{-1} \text { and }(a \delta)^{-1} F(a \delta)=(b \delta)^{-1} F(b \delta)
$$

for all idempotents $F$ in $S / \delta$. Now $F=f \delta$ for some $f \in E$, and for any $a^{\prime} \in V(a),(a \delta)^{-1}=\left(a^{\prime} \delta\right)$ since $\delta$ is an inverse semigroup congruence. So $a \tau b \Leftrightarrow(a \delta)(f \delta)\left(a^{\prime} \delta\right)=(b \delta)(f \delta)\left(b^{\prime} \delta\right)$ and 
$\left(a^{\prime} \delta\right)(f \delta)(a \delta)=\left(b^{\prime} \delta\right)(f \delta)(b \delta) \Leftrightarrow a f a^{\prime} \delta b f b^{\prime}$ and $a^{\prime} f a \delta b^{\prime} f b$ for some (all) $a^{\prime} \in V(a)$ and $b^{\prime} \in V(b)$ and all $f \in E$.

We can now prove the following result.

LEMMA 4.5. Let $S$ be an orthodox semigroup satisfying

$$
h, f \in E, h \leqq f \mathscr{H} a, a^{-1} \in V(a) \cap H_{a} \text {, and } a h a^{-1} \in V(h) \text { imply } a h a^{-1}=h .
$$

Then on $S, \mu \vee \delta=\tau$, the largest element of $\delta \theta$.

Proof. It suffices to show that $a \tau e$ implies $a \mu \vee \delta e$. To motivate the proof, note that if $\mu \vee \delta=\tau$ then, by Lemma 3.1, $\mu \circ \delta=\tau$, and thus $a \tau e$ implies the existence of an $x$ such that $a \mu x \delta e$. Then $x \delta e$ implies $x \in E$, and $a \mu x$ implies $a \mathscr{H} x$, so that $x$ must be the identity of $H_{a}$.

Assume that $a \tau e$. We first show that $H_{a}$ is a group with identity $f$, and $a \tau f$. Since $\tau$ is an inverse semigroup congruence, $a \tau e$ implies $a^{\prime} \tau e$ for each $a^{\prime} \in V(a)$. Thus $a a^{\prime} \tau e$ and $a^{\prime} a \tau e$. Now $a a^{\prime}$ and $a^{\prime} a$ are idempotents which are inverses of one another ([13]), so $\left(a a^{\prime}\right)\left(a^{\prime} a\right)$ is an idempotent $f$ in $H_{a}$ and $H_{a}$ is a group ([1, p. 58-9]). Moreover, $f=\left(a a^{\prime}\right)\left(a^{\prime} a\right) \tau e \tau a$.

From this we deduce that since $\tau \theta \delta$ we have $e \delta f$. From the discussion preceding the statement of the lemma, we know that $a \tau f$ implies $a h a^{\prime} \delta f h f \delta a^{\prime} h a$ for all $h \in E$ and $a^{\prime} \in V(a)$. Let $g \in E$ and $a^{-1} \in V(a) \cap H_{a}$. Then the idempotent $h=f g f$ is below $f$ and satisfies $a h a^{-1}=$ $a f g f a^{-1}=a g a^{-1} \delta f g f=h$, and similarly $a^{-1} h a \delta h$. But $a h a^{-1} \delta h$ implies $a h a^{-1} \in V(h)$ by Result 1.5, so we have $h \leqq f \mathscr{H} a \mathscr{H} a^{-1}$ and $a h a^{-1} \in V(h)$ and $a^{-1} h a \in V(h)$. So the hypotheses of (*) are satisfied, and we conclude that $a h a^{-1}=h=a^{-1} h a$, i.e. $a g a^{-1}=f g f=a^{-1} g a$ for all $g \in E$. By Meakin's characterization of $\mu$ we have $a \mu f$, and, combined with $e \delta f$, this means $a \mu \vee \delta e$. The proof is complete.

We discuss below various classes of orthodox semigroups in which (*) is satisfied. An example of one in which it is not satisfied is the following.

Let

$$
T=\left\langle a, e, f: a^{2}=e, e^{2}=e, f^{2}=f, e a=a e=a, e f=f e=f,(a f)(a f)=a f,(f a)(f a)=f a\right\rangle .
$$

Then $T$ is a six element orthodox semigroup whose Green's relations are described by the following diagram.

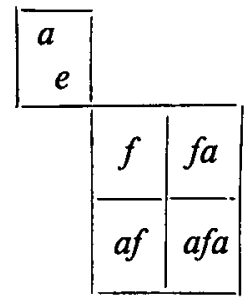

Here $f \leqq e \mathscr{H} a$ and $a f a \in V(f)$ but $a f a \neq f$. There are four congruences on $T$ :

$\rho=\{(f, f a)\}^{*}$ collapses the rows of the rectangular band;

$\lambda=\{(f, a f)\}^{*}$ collapses the columns of the rectangular band;

$\delta=\rho \vee \lambda$ identifies all elements of the rectangular band;

$\tau=\{(e, a)\}^{*}$ has two classes, one of which is $\{e, a\}$.

Since $\mu=0, \mu \vee \delta=\delta$. However $\tau$ is the largest element of $\delta \theta$, and $\tau \neq \delta$. 
THEOREM 4.6. Let $S$ be an orthodox semigroup such that each homomorphic image of $S$ satisfies condition (*). Then $\Lambda(S)$ is a union of products of $\theta$-classes and $\Phi$-classes in the following sense:

$\Lambda(S)=\cup\{[\bar{\beta}, \tau]: \tau$ and $\beta$ are the maximum and minimum of $a \theta$-class above $\delta\}$, and each $[\bar{\beta}, \tau]$ is isomorphic to $\bar{\beta} \theta \times \bar{\beta} \Phi$.

Proof. Let $\rho \in \Lambda(S)$ and let $\tau$ and $\beta$ be, respectively, the maximum and minimum elements of $(\rho \vee \delta) \theta$. It is easily seen that $[\bar{\beta}, \tau]$ is a union of $\Phi$-classes and that

$\{[\bar{\beta}, \tau]: \tau$ and $\beta$ are the maximum and minimum of a $\theta$-class above $\delta\}$

is a partition of $\Lambda(S)$. We show that $[\bar{\beta}, \tau]$ is isomorphic to $\bar{\beta} \theta \times \bar{\beta} \Phi$.

From the remarks preceding the previous lemma, $\bar{\beta} \theta$ is isomorphic to $\Lambda(\mu(S / \bar{\beta}))$ under the map $\gamma \rightarrow \gamma / \bar{\beta}$; and $\mu(S / \bar{\beta})=\bar{\tau} / \bar{\beta}$ where $\bar{\tau}$ is the maximum of $\bar{\beta} \theta$. We claim that $\bar{\beta} \Phi$ is isomorphic to the lattice $\Lambda(\delta(S / \bar{\beta})$ ) of congruences on $S / \bar{\beta}$ below $\delta(S / \bar{\beta})$ under the same map $\sigma \rightarrow \sigma / \bar{\beta}$. For

$$
\lambda \in \bar{\beta} \Phi \Leftrightarrow \bar{\beta} \leqq \lambda \leqq \bar{\beta} \vee \delta=\beta \Leftrightarrow \mathbf{0}(S / \bar{\beta})=\bar{\beta} / \bar{\beta} \leqq \lambda / \bar{\beta} \leqq(\bar{\beta} \vee \delta) / \bar{\beta}=\delta(S / \bar{\beta}) .
$$

Now the map $\gamma \rightarrow \gamma / \bar{\beta}$ sends $[\bar{\beta}, \tau]$ to $\{\alpha \in \Lambda(S / \bar{\beta}): 0 \leqq \alpha \leqq \tau / \bar{\beta}\}$. Since $S / \bar{\beta}$ satisfies $(*)$ and $\delta(S / \bar{\beta})=(\delta \vee \bar{\beta}) / \bar{\beta}=\beta / \bar{\beta}$, the top of the $\theta$-class of $\delta(S / \bar{\beta})=\beta / \bar{\beta}$ is $\mu(S / \bar{\beta}) \vee \delta(S / \bar{\beta})=\bar{\tau} / \bar{\beta} \vee$ $\beta / \bar{\beta}=(\bar{\tau} \vee \beta) / \bar{\beta}$. Thus $\mu(S / \beta)=$ maximum element of $(\beta / \bar{\beta}) \theta] /(\beta / \bar{\beta})=(\bar{\tau} \vee \beta) / \beta$. However, we also have $\mu(S / \beta)=\tau / \beta$, so that $\tau / \beta=(\bar{\tau} \vee \beta) / \beta$, and hence $\tau=\bar{\tau} \vee \beta$. We conclude that

$$
\begin{aligned}
& \{\alpha \in \Lambda(S / \bar{\beta}): 0 \leqq \alpha \leqq \tau / \bar{\beta}\} \\
& \quad=\{\alpha \in \Lambda(S / \bar{\beta}): \mathbf{0} \leqq \alpha \leqq \bar{\tau} \vee \beta) / \bar{\beta}=\mu(S / \bar{\beta}) \vee \delta(S / \bar{\beta})\} \\
& \quad=\Lambda(\mu(S / \bar{\beta}) \vee \delta(S / \bar{\beta})) .
\end{aligned}
$$

We have thus shown that the mapping $\gamma \rightarrow \gamma / \bar{\beta}$ is an isomorphism both from $[\bar{\beta}, \tau]$ onto $\Lambda(\mu(S / \bar{\beta}) \vee \delta(S / \bar{\beta}))$ and from $\bar{\beta} \theta \times \bar{\beta} \Phi$ onto $\Lambda(\mu(S / \bar{\beta})) \times \Lambda(\delta(S / \bar{\beta}))$. Since the two ranges are isomorphic by Theorem 4.4, the two domains are isomorphic, and the proof is complete.

COROLlaRY 4.7. Let $S$ be an orthodox semigroup satisfying either of the following conditions:

(a) $S$ is a band of groups;

(b) $E$ is naturally ordered ( $e \leqq f$ and $g \leqq h$ imply $e g \leqq f h$ for all $e, f, g, h \in E$ ).

Then each homomorphic image of $S$ satisfies (*), and hence $\Lambda(S)$ is a union of products of $\theta$ classes and $\Phi$-classes in the sense of Theorem 4.6.

Proof. Let $S$ be an orthodox semigroup and let $f, h \in E$ with $h \leqq f \mathscr{H} a, a^{-1} \in V(a) \cap H_{a}$, and $a h a^{-1} \in V(h)$.

(a) If $S$ is a band of groups, then $\mathscr{H}$ is a congruence. Hence $h \leqq f \mathscr{H} a$ and $a^{-1} \in H_{a}$ imply $h=$ fhf $\mathscr{H} a h a^{-1}$. However, $a h a^{-1} \in E\left(\left[13\right.\right.$, Lemma 1.4]) and we conclude that $h=a h a^{-1}$.

Spitznagel [17] has shown that any homomorphic image of a band of groups is again a band of groups. Hence each homomorphic image of $S$ satisfies (*).

(b) If $E$ is naturally ordered we compute that $h=h a h a^{-1} h \leqq f a h a^{-1} h=a h a^{-1} h$. Easily we have $a h a^{-1} \leqq f$, so $a h a^{-1} h \leqq f h=h$. These together yield $h=a h a^{-1} h$. A similar argument 
shows $h=h a h a^{-1}$. Hence $a h a^{-1}=\left(a h a^{-1}\right) h\left(a h a^{-1}\right)=h a h a^{-1}=h$, and so $S$ satisfies (*). Howie [5] has shown that the class of naturally ordered bands is a subvariety of the variety of bands, and hence that any homomorphic image of a naturally ordered band is again naturally ordered. Combining this with Result 1.4 we conclude that any homomorphic image of $S$ also has a naturally ordered band of idempotents and hence satisfies condition (*).

Remark. The results of this section yield a method for computing all the congruences on (and hence all homomorphic images of) an orthodox semigroup satisfying the hypotheses of Theorem 4.6 if one assumes that the congruences on the inverse semigroup $S / \delta$ are " known". One computes $I(S)$ by using the inverse of the isomorphism $\rho \rightarrow \rho / \delta$ of this set onto $\Lambda(S / \delta)$. By computing the minimum element $\beta$ of each $\theta$-class and then computing $\beta \Phi$, one can then compute $\beta \theta \times \beta \Phi$. These computations may be simplified by noting that if we define $\beta=\{(e f e, e): e \beta f\}^{*}$ then $\beta=\bar{\beta}$. For since $\bar{\beta} \vee \delta=\beta$, by Lemma $2.2\{(e f e, e): e \beta f\} \subseteq \bar{\beta}$, and hence $\beta \leqq \bar{\beta}$. Thus $\beta \vee \delta \leqq \bar{\beta} \vee \delta=\beta$. However, if $e \beta f$ then $e f e \beta e$ and $f e f \beta f$, so by Lemma $2.2 e(\beta \vee \delta) f$. We conclude that $(\beta \vee \delta) \theta \beta$; however since $\beta$ is the minimum of its $\theta$-class, $\beta \vee \delta=\beta$. This means $\bar{\beta} \leqq \beta$, and we conclude $\bar{\beta}=\beta$. It is because of this description of $\bar{\beta}$ that Theorem 4.6 was stated in terms of the minimum elements in $\theta$-classes; in fact, one can show that if $\tau \in \beta \theta$ (and the hypotheses of Theorem 4.6 are satisfied) then $\beta \theta \times \beta \Phi$ is isomorphic to $\bar{\tau} \theta \times \tau \Phi$.

5. Applications and related results. Scheiblich has shown [15] that any congruence $\rho$ on an inverse semigroup is completely determined by its restriction $\rho \cap(E \times E)(\theta$-class $)$ and $\operatorname{ker} \rho$. Green [2] has given an alternative approach to the result. We now prove this result for orthodox semigroups.

THEOREM 5.1. Let $S$ be an orthodox semigroup and let $\rho, \tau \in \Lambda(S)$. Then $\rho=\tau$ iff $\rho \theta \tau$ and $\operatorname{ker} \rho=\operatorname{ker} \tau$.

Proof. If $\rho \theta \tau$, then $(\rho \vee \delta) \theta(\tau \vee \delta)$ since $\theta$ is a congruence. If $\operatorname{ker} \rho=\operatorname{ker} \tau$, then $\operatorname{ker}(\rho \vee \delta)$ $=\operatorname{ker}(\tau \vee \delta)$ by Lemma 2.1. Thus in $\Lambda(S / \delta),[(\rho \vee \delta) / \delta] \theta[(\tau \vee \delta) / \delta]$ and $\operatorname{ker}((\rho \vee \delta) / \delta)=$ $\operatorname{ker}((\tau \vee \delta) / \delta)$. By Scheiblich's result we then have $(\rho \vee \delta) / \delta=(\tau \vee \delta) / \delta$, so $\rho \vee \delta=\tau \vee \delta$. Since $\rho \theta \tau$ by assumption, Lemma 3.3 implies $\rho=\tau$, and the proof is complete.

Howie and Lallement [7] have shown the existence of a minimum orthodox congruence $\lambda$ on a regular semigroup $T$. We will now consider extending the results of the previous sections to $\Omega(T)=\{$ orthodox congruences on $T\}=\Lambda(T) \vee \lambda$.

Lemma 5.2. Let $T$ be a regular semigroup and let $\rho, \tau \in \Lambda(T)$ be such that $\rho \theta \tau$. Then $\rho \in I(T)$ iff $\tau \in I(T)$.

Proof. We first claim that for an orthodox semigroup $S, \rho \theta \tau$ and $\rho \in I(S)$ imply $\tau \in I(S)$. For $\rho \in I(S) \Leftrightarrow e f \rho f e$ for all $e, f \in E \Leftrightarrow e f \tau f e$ for all $e, f \in E$ (since $\rho \theta \tau$ and $e f, f e \in E) \Leftrightarrow \tau \in I(S)$.

Next we claim that if $\rho, \tau \in \Lambda(T)$ and $\rho \theta \tau$ with $\rho \in I(T)$ then $\tau \in \Omega(T)$. This can be proved by showing that if $F$ and $G$ are idempotent $\tau$-classes then $F G$ contains an idempotent. Now $F=f \tau$ and $G=g \tau$ for some $f, g \in E$ by Result 1.4. Howie and Lallement [7] have shown that we can always find an idempotent inverse $e$ for $f g$. Then $e f g=h_{1}$ and $f g e=h_{2}$ are idempotents. 
Now $e=e(f g) e \delta e(e f g)=e f g=h_{1}$, since idempotents commute in $T / \delta$. Similarly $e \delta h_{2}$. Since $\delta \leqq \rho$, we have $e \rho h_{1}$ and $e \rho h_{2}$, so $e \tau h_{1}$ and $e \tau h_{2}$. We conclude that $e=e^{2} \tau h_{2} h_{1}=$ $(f g e)(e f g)=f g$, and the second claim is proved.

Finally to prove the lemma we note that if $\rho, \tau \in \Lambda(T)$ and $\delta \leqq \rho \theta \tau$ then, by the above, $\tau \geqq \lambda$. Hence $\tau / \lambda$ is defined, and $\rho / \lambda \theta \tau / \lambda$. Moreover $\rho / \lambda \geqq \delta / \lambda=\delta(S / \lambda)$ since $\delta \geqq \lambda$. Thus by the first claim $\tau / \lambda$ is an inverse semigroup congruence (on the orthodox semigroup $S / \lambda$ ) and hence $\tau / \lambda \geqq \delta / \lambda$. We conclude that $\tau \geqq \delta$. Interchanging the roles of $\rho$ and $\tau$ yields a corresponding result, and the lemma is proved.

The above result says that the sublattice $I(S)$ of $\Lambda(S)$ is $\theta$-saturated, a fact which can be inferred, for bands of groups, from Proposition 4.1 of [16]. One should note that $\Omega(S)$ is not in general $\theta$-saturated; for in $[7, \mathrm{p} .153]$ it is shown that the minimum band congruence $\beta$ is the top of the $\theta$-class of the minimum band of groups congruence $\pi$ and $\pi \leqq \pi \vee \lambda \leqq \beta$, but $\pi$ need not be in $\Omega(S)$.

Since $I(S) \subseteq \Omega(S)$, it follows than any $\rho \in I(S)$ can be written in the form $\rho=\tau / \lambda$ for some $\tau \in \Omega(S)$. Consequently $\delta(S / \lambda)=\delta(S) / \lambda$ and $S / \delta=(S / \lambda) /(\delta / \lambda)$.

THEOREM 5.3. Let $S$ be a regular semigroup. The map $\rho \rightarrow(\rho \theta,(\rho \vee \delta) / \delta)$ is an embedding of $\Omega(S)$ into $\Omega(S) / \theta \times \Lambda(S / \delta)$.

Proof. Note that first $\rho \rightarrow \rho / \lambda$ is an isomorphism of $\Omega(S)$ to $\Lambda(S / \lambda)$. By Corollary 3.5 the map $\rho / \lambda \rightarrow((\rho / \lambda) \theta,((\rho / \lambda) \vee \delta(S / \lambda)) / \delta(S / \lambda))$ is an embedding. Now $\rho / \lambda \theta \tau / \lambda \Leftrightarrow \rho \theta \tau$, and $((\rho / \lambda) \vee \delta(S / \lambda)) / \delta(S / \lambda)=((\rho / \lambda) \vee(\delta / \lambda)) /(\delta / \lambda)=(\rho \vee \delta) / \delta$. It thus follows that $\rho \rightarrow(\rho \theta$, $(\rho \vee \delta) / \delta)$ is an embedding.

The definition of the relation $\Phi$ in Section 3 can be extended to $\Omega(S)$ : for $\rho, \tau \in \Omega(S)$, $\rho \Phi \tau \Leftrightarrow \rho \vee \delta=\tau \vee \delta$. Since

$$
\rho \Phi \tau \Leftrightarrow \rho / \lambda \vee \delta / \lambda=\tau / \lambda \vee \delta / \lambda \Leftrightarrow \rho / \lambda \vee \delta(S / \lambda)=\tau / \lambda \vee \delta(S / \lambda)
$$

it follows from the results of Section 2 that $\Phi$ is a congruence on $\Omega(S)$. Using Theorem 3.7 and techniques like those in the previous theorem, one can prove the following (we omit the details).

THEOREM 5.4. Let $S$ be a regular semigroup. Then

(a) $\Upsilon(\rho)=(\rho \wedge \delta, \rho \vee \delta)$, when restricted to $\Omega(S)$, is one-to-one;

(b) if $\Omega(S)$ is $\Phi$-modular, then $\Upsilon$ is a lattice embedding of $\Omega(S)$ into $[\lambda, \delta]$.

The function $\Upsilon$ above can be defined on all of $\Lambda(S)$; however, it need not be one-to-one, as the following example shows.

Let $G=\{e, a, b, a b\}$ be the Klein Four-group, and let $I=\{1,2\}$. Let $S$ be the $I \times I$ Rees matrix semigroup over $G^{0}$ with sandwich matrix $P$ having all entries $e$ except for one entry $a b$. One computes that $\Lambda(S) \wedge \mathscr{H}$ has three congruences other than $\mathbf{0}_{s}$ and $\mathscr{H}$. Call these $\lambda$ (for one is the minimum orthodox congruence), $\alpha$ and $\beta$. None of these is comparable to any other, and one can show that $\Upsilon(\alpha)=\left(\mathbf{0}_{S}, \mathbf{1}_{S}\right)=\Upsilon(\beta)$. 

in $[\mathbf{1 8}]$.

Note added in proof. Theorem 5.1 has been obtained independently by Ruth Fergenbaum

\section{REFERENCES}

1. A. H. Clifford and G. B. Preston, The algebraic theory of semigroups, Vols. 1 and 2, Amer. Math. Soc. Mathematical Surveys No. 7 (Providence, R.I., 1961, 1967). $141-152$.

2. D. G. Green, The lattice of congruences on an inverse semigroup, Pacific J. Math. 57 (1975),

3. T. E. Hall, On regular semigroups whose idempotents form a subsemigroup. Bull. Austral. Math. Soc. 1 (1969), 195-208.

4. T. E. Hall, On the lattice of congruences on a regular semigroup, Bull. Austral. Math. Soc. 1 (1969), 231-235.

5. J. M. Howie, Naturally ordered bands, Glasgow Math. J. 8 (1967), 55-58.

6. J. M. Howie, The maximum idempotent-separating congruence on an inverse semigroup, Proc. Edinburgh Math. Soc. (2) 14 (1964-5), 71-79.

7. J. M. Howie and G. Lallement, Certain fundamental congruences on a regular semigroup, Proc. Glasgow Math. Assoc. 7 (1966), 145-159.

8. K. M. Kapp and H. Schneider, Completely 0-simple semigroups (New York, 1969).

9. G. Lallement, Congruences et equivalences de Green sur un demi-groupe regulier, C. R. Acad. Sci. Sér A, 262 (1966), 613-616.

10. J. Meakin, Congruences on orthodox semigroups, J. Austral. Math. Soc. 12 (1971), 323-341.

11. W. D. Munn, A certain sublattice of the lattice of congruences on a regular semigroup, Proc. Cambridge Philos. Soc. 60 (1964), 385-391.

12. G. B. Preston, Congruences on completely simple semigroups, Proc. London Math. Soc. (3) 11 (1961), 557-576.

13. N. R. Reilly and H. E. Scheiblich, Congruences on regular semigroups, Pacific J. Math. 23 (1967), 349-360.

14. H. E. Scheiblich, Certain congruence and quotient lattices related to completely 0 -simple and primitive regular semigroups, Glasgow Math. J. 10 (1969), 21-24.

15. H. E. Scheiblich, Kernels of inverse semigroup homomorphisms, J. Austral. Math. Soc. 18 (1974), 289-292. 187-197.

16. C. Spitznagel, The lattice of congruences on a band of groups, Glasgow Math. J. 14 (1973),

17. C. Spitznagel, $\theta$-modular bands of groups, Trans. Amer. Math. Soc. 177 (1973), 469-482.

18. Ruth Fergenbaum, Kernels of orthodox semigroup homomorphisms, J. Austral. Math. Soc. Ser. $A 22$ (1976), 234-245.

Department of Mathematics

UNIVERSITY OF KENTUCKY

LEXINGTON, KeNTUCKY 40506

U.S.A.
Department of Mathematics

UNIVERSITY OF LOUISVILLE

LOUISVILLE, KENTUCKY 40208 U.S.A. 